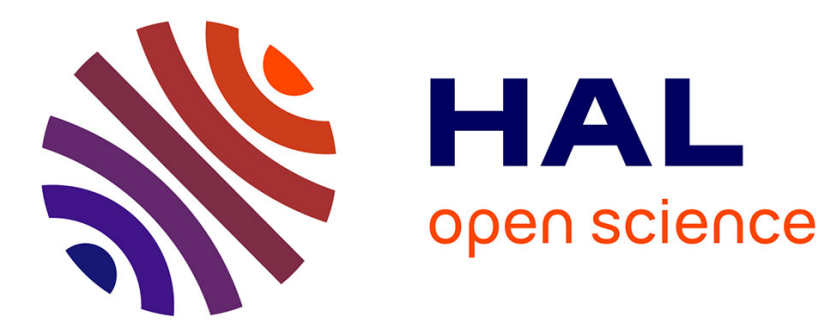

\title{
Clinique de l'hospitalisation involontaire
}

\author{
Myriam Guedj, Marjolaine Henault, Amandine Pinault
}

\section{To cite this version:}

Myriam Guedj, Marjolaine Henault, Amandine Pinault. Clinique de l'hospitalisation involontaire: Un exemple de recherche en psychologie clinique de la santé. Le Journal des psychologues, 2008, 260, pp.48-51. 10.3917/jdp.260.0048 . hal-01953106

\section{HAL Id: hal-01953106 \\ https://hal-univ-tlse2.archives-ouvertes.fr/hal-01953106}

Submitted on 6 Jul 2021

HAL is a multi-disciplinary open access archive for the deposit and dissemination of scientific research documents, whether they are published or not. The documents may come from teaching and research institutions in France or abroad, or from public or private research centers.
L'archive ouverte pluridisciplinaire HAL, est destinée au dépôt et à la diffusion de documents scientifiques de niveau recherche, publiés ou non, émanant des établissements d'enseignement et de recherche français ou étrangers, des laboratoires publics ou privés. 


\section{Myriam Guedj}

Psychologue

Postdoctorante en psychologie de la santé

INSERM U558, département d'épidémiologie et de santé publique, université Toulouse-LeMirail

\section{Avec la collaboration de}

\section{Marjolaine Henault}

\section{Amandine Pinault}

Étudiantes en master de psychologie

CERPP, OCTOGONE, université Toulouse-Le-Mirail

\section{Clinique de l'hospitalisation involontaire}

\section{Un exemple de recherche en psychologie clinique de la santé}

Notre recherche en psychologie clinique de la santé porte sur la clinique de l'hospitalisation involontaire. Nous avons choisi d'aborder une thématique clinique à travers l'analyse de l'acceptabilité de cette décision, et notamment le jugement du risque représenté par le patient. (L'hospitalisation involontaire est décidée lorsqu'une personne n'est pas en mesure d'exercer son consentement, mais que son hospitalisation est nécessaire.) Cette pratique concerne le plus souvent une personne présentant un trouble mental qui l'empêche de se prendre en charge ou qui induit un comportement dangereux pour elle-même ou son entourage. De manière générale, la notion de dangerosité sert à caractériser une situation, une chose ou une personne présentant un danger pour son environnement physique ou humain, ou encore pour elle-même. Elle réfère au risque, c'est-à-dire à la potentialité, la possibilité ou la probabilité d'une issue néfaste. Cependant, la dangerosité et son substrat - le danger - sont des concepts complexes que certains modèles cherchent à cerner et à mesurer (Kouabenan et al., 2006), bien que le danger (et le risque) soit une donnée complexe que l'on peut difficilement définir, identifier et, surtout, évaluer en situation concrète (Appelbaum et al., 2000). 
Après avoir précisé le contexte juridique de l'hospitalisation sans consentement, nous proposerons une revue détaillée de la littérature, afin de préciser les critères habituellement utilisés pour évaluer la dangerosité des patients. Nous présenterons, par la suite, notre projet de recherche, projet dont le but est d'analyser l'acceptabilité de l'hospitalisation involontaire chez le grand public, concerné par ce type de décision, notamment en tant que famille de patient présentant des troubles mentaux, mais également en tant que proche ou professionnel confronté à ce type de situation.

\section{Contexte juridique}

La clinique de l'hospitalisation involontaire est très liée, en France, à la notion de danger. En effet, celui-ci revêt une place centrale en droit français, car la législation psychiatrique dérive directement du traitement des malades mentaux criminels et délinquants. L'ancien article L. 326 du Code de la santé publique définissait ainsi l'aliénation mentale par référence à la fréquence des actes ou accès de démence de la personne. La réforme du 27 juin 1990 a modifié la notion de placement volontaire en hospitalisation à la demande d'un tiers (HDT) et hospitalisation d'office (HO), qui renvoient à des situations différentes.

L'hospitalisation à la demande d'un tiers suppose, d'une part, que l'état de santé de la personne nécessite, du point de vue médical, la mise en œuvre de traitements particuliers, d'autre part, que ces traitements ne puissent être administrés que dans un cadre hospitalier, sous surveillance médicale constante. Enfin, il faut encore que les troubles de la personne rendent impossible son consentement et que le danger pour elle-même caractérise un péril imminent. Les dispositions relatives à l'hospitalisation d'office, telles qu'elles résultent de la réforme du 27 juin 1990, confient des pouvoirs aux préfets des départements, considérant des situations d'une tout autre nature et un danger tout à fait particulier : il faut que les troubles mentaux de la personne « compromettent l'ordre public ou la sûreté des personnes ». Le législateur a tendu à réserver l'hospitalisation d'office au cas du danger pour l'ordre public et la sûreté d'autrui, bien que la référence à « la sûreté des personnes » ne permette pas d'exclure catégoriquement le danger pour soi-même (Bernardet, 2003).

\section{Une évaluation de la dangerosité qui procède d'une lecture clinique}

On prête beaucoup aux psychiatres en matière de prédiction de dangerosité, et bien plus que l'état des connaissances ne l'autorise (Van Gijseghem, 1996). Cependant, les cliniciens 
engagés dans le champ des troubles mentaux graves et persistants, tout comme ceux qui interviennent principalement dans le champ des troubles de la personnalité, sont régulièrement appelés à évaluer - notamment dans la décision d'hospitalisation - le risque de comportements violents dont pourraient se rendre responsables leurs patients. Les premières études qui ont cherché à estimer la capacité des cliniciens de prédire la « dangerosité » ont brossé un tableau assez pessimiste de la capacité des cliniciens de ce faire, pessimisme qui a prévalu au cours des années soixante à quatre-vingt (Coté, 2001). De plus, la relation entre le diagnostic psychiatrique et la violence est controversée. La littérature contient beaucoup de résultats contradictoires. Beaucoup de troubles mentaux sont épisodiques, leur influence sur le risque de violence pouvant seulement apparaître quand le patient est en crise. J. Monahan (1992) a comparé les taux de violence par rapport à la population générale. Il trouve que la prévalence de violence est multipliée par cinq chez les personnes qui répondent aux critères de l'axe I du DSM-III, que la prévalence de violence est identique chez les personnes atteintes de schizophrénie, dépression ou trouble bipolaire et, enfin, que la prévalence de violence est multipliée par douze chez les personnes diagnostiquées alcooliques et par seize chez les personnes diagnostiquées comme abusant de drogue. Parmi les patients gravement malades vus dans un contexte civil (nonhospitalisation), les diagnostics de schizophrénie, de désordre bipolaire, d'épisode maniaque et de psychose sont associés à un risque élevé de violence à court terme (Tardiff, 1996). Plus précisément, il semble que les symptômes aigus prédisent la violence à court terme (Link et al., 1992). Ceux-ci incluent excitation, agitation, hostilité, suspicion et perturbation de la pensée (désorganisation conceptuelle, hallucinations et pensées inhabituelles). B. G. Link, A. Stueve et J. Phelan (1998) ont d'ailleurs identifié des symptômes psychotiques marqués par des symptômes « de priorité de contrôle et de menace » qui augmenteraient le risque de violence. Ce modèle de symptômes se rapporte au sentiment du patient d'être menacé et de ne pas contrôler ses actes et pensées.

La clinique suggère également que les hallucinations de contrôle augmentent le risque de violence, bien que la littérature empirique n'ait pas uniformément soutenu cette hypothèse (Appelbaum et al., 2000).

L'association entre les symptômes et le risque de violence identifiée dans les études montre donc l'utilité d'un examen mental complet dans l'évaluation du potentiel violent d'une personne. En effet, un individu peut ne pas menacer explicitement d'être violent, mais présenter des symptômes qui augmentent le risque de violence (délire paranoïaque, par exemple). Plus généralement, l'association entre la psychopathologie et la violence souligne 
l'importance du diagnostic, y compris l'évaluation de l'histoire de la maladie actuelle, de l'histoire psychiatrique et de l'examen mental. C'est pourquoi, même si le psychiatre n'est pas toujours le mieux placé pour évaluer la dangerosité de son patient, il est dans une position où il détient un savoir sur son patient, savoir qui peut lui donner certaines indications sur la violence interne de celui-ci. À la suite du pessimisme des années quatrevingt, les pressions légales, notamment liées aux modifications des lois sur l'hospitalisation involontaire d'une personne atteinte d'un trouble mental, ont conduit à l'émergence $\mathrm{d}^{\prime}$ instruments pour l'évaluation $\mathrm{du}$ « risque ». Il ne s'agit plus d'évaluer si le patient est dangereux ou non, mais d'estimer le risque de comportements violents pour un individu donné, dans un contexte donné, et selon un temps donné.

\section{Les facteurs de risque de comportements violents}

En général, les recherches actuelles penchent beaucoup plus vers l'importance des facteurs individuels que l'importance des facteurs communautaires ou sociétaux dans la probabilité des comportements violents. De fait, on s'entend de plus en plus pour dire que la conjugaison de facteurs personnels, circonstanciels, sociaux et culturels débouche sur la violence (Heise, 1998).

Selon l'étude de McCauley et al. (1995), sur neuf facteurs de risque, quand le patient n'en présente qu'un, le risque de violence s'élève à 1,2 \%. En revanche, quand six à sept facteurs de risque sont présents, la probabilité de devenir violent s'élève à 70,4\%. La violence est plus à même de se manifester chez un homme jeune, de classe sociale basse, avec un diagnostic d'abus de substance et manifestant un trouble mental majeur (Swanson, 1994).

Âge

De nombreuses études ont constaté que la violence est plus fréquente parmi des personnes plus jeunes (McCauley et al., 1995). Cependant, l'âge semble agir avec d'autres facteurs de risque. Par exemple, parmi les plus jeunes patients, des diagnostics tels que la schizophrénie aiguë ou la manie aiguë sont associés au risque de violence (Tardiff, 1996). Parmi les patients plus âgés, il y a une plus grande représentation des troubles mentaux organiques tels que la démence parmi les patients qui deviennent violents (Kalunian et al., 1990).

La valeur prédictive de l'âge comme indicateur de risque de violence semble également changer en fonction de la phase de la maladie. Parmi les populations étudiées, les personnes plus jeunes s'avèrent plus violentes (Swanson et al., 1990). Cependant, parmi les patients 
atteints de maladie mentale grave, dont la conscience est suffisamment altérée pour justifier une hospitalisation, les facteurs cliniques, tels que le diagnostic et les symptômes, prennent le pas sur les facteurs démographiques, tels que l'âge, pour prédire de futurs comportements.

\section{Sexe}

Traditionnellement, les hommes ont été considérés comme étant beaucoup plus violents que les femmes (Monahan, 1981). Cependant, les recherches récentes réalisées auprès de patients psychiatriques ont suggéré que les passages à l'acte étaient réalisés avec plus de violence chez les femmes. Par exemple, dans une étude réalisée auprès de personnes hospitalisées en psychiatrie, les femmes étaient réellement plus agressives physiquement que les hommes, bien que les hommes se soient engagés dans plus de conduites impliquant des comportements violents (par exemple, menaces, dégâts matériels et agressions verbales). De même, dans une autre étude portant sur des patients récemment admis dans un service d'urgence psychiatrique, des taux comparables de violence ont été trouvés parmi les patients féminins et masculins, bien que ces derniers se soient engagés sous des formes plus graves de violence que les femmes (Newhill et al., 1995).

\section{Antécédents de violence intrafamiliale}

De nombreuses études ont prouvé que le fait d'avoir été victime d'abus dans l'enfance ou d'avoir grandi dans une maison violente augmente le risque d'une personne de devenir violente au cours de sa vie (Black et al., 1999). Un antécédent de violence s'est avéré le meilleur prédicteur possible du comportement violent (Bailey et al., 1997). L'évaluation de la fréquence de la violence passée est utile, des études portant sur des patients suivis par les hôpitaux en soins externes et internes ont prouvé qu'une grande partie d'un petit nombre d'individus commet une grande partie des comportements violents, et ce, de façon répétitive (Gardner et al., 1996).

\section{Adhérence au traitement}

Le traitement qui est associé au potentiel violent peut réduire le risque de violence des patients. Ainsi, J. A. Yesavage et al. (1983) ont montré un rapport inverse entre les niveaux de sérum neuroleptique d'un groupe de patients schizophrènes hospitalisés et leur niveau d'agression. Il est possible que des différences entre la violence au sein de la communauté et la violence au sein de l'hôpital soient dues au point auquel les patients adhèrent à leur 
traitement. Par exemple, certains patients n'agressent personne à l'hôpital quand ils reçoivent le traitement pharmacologique approprié, mais, lorsqu'ils arrêtent leur traitement à leur sortie de l'hôpital, ils deviennent violents au moment de la réapparition de leurs symptômes (Johnson et al., 1983).

\section{L'acceptabilité de la décision d'hospitalisation involontaire}

Les facteurs de risque cités précédemment, bien connus des professionnels de santé, leur permettent d'évaluer la nécessité de soin d'un patient ou son éventuelle hospitalisation. D'ailleurs, selon D. J. Luchins et al. (2004), les décisions d'hospitalisation involontaire augmentent avec le niveau de risque d'atteinte (hétéro ou autoagressif) et varient en fonction du diagnostic psychiatrique : schizophrénie, PMD, toxicomanie. De la même façon, d'autres recherches portant sur le rôle de la violence dans les décisions d'hospitalisation dans les services d'urgence psychiatriques (McNiel et al., 1992) montrent que bien que les patients violents soient vraisemblablement plus hospitalisés que les patients non violents, des variables telles que le diagnostic et la gravité globale de l'atteinte psychique semblent être plus importantes que le comportement violent dans les prédictions de décision d'hospitalisation. Les psychiatres ont plutôt hospitalisé les patients les plus gravement perturbés présentant des diagnostics tels que des troubles schizophrènes et maniaques pour lesquels un arsenal thérapeutique est largement admis. D'autres auteurs ont, quant à eux, mis en évidence trois liens fondamentaux sous-tendant l'estimation globale du risque représenté par un patient, entraînant de ce fait une hospitalisation. Deux sont relatifs aux caractéristiques du clinicien : la proportion de patients qu'a hospitalisés le clinicien dans les trois mois précédents et le service dans lequel l'évaluation s'est produite (service d'urgence ou unité mobile de crise). La disponibilité des lits d'hospitalisation était également un facteur prédictif significatif de l'hospitalisation d'un patient. Aucune caractéristique du patient, y compris le diagnostic, le sexe, l'âge ou le statut d'assurance, n'a été liée à la décision d'hospitalisation dans cette recherche (Engleman et al., 1998). Par ailleurs, la recherche comparative entre les populations anglaises et allemandes, menée par P. Lepping et al. (2004), tend à montrer que les attitudes concernant les décisions d'hospitalisation involontaire sont équivalentes entre les médecins, les infirmières et le grand public, à l'exception des praticiens non psychiatres qui n'activent pas systématiquement le processus de l'hospitalisation. Cette recherche, rare au niveau international, pose la question du poids 
de l'opinion du grand public dans la décision d'hospitaliser des patients atteints de troubles mentaux.

Cette question pourrait paraître inutile, mais à y regarder de plus près, elle est d'une grande pertinence. En effet, que connaît-on de l'entourage des patients psychiatriques ? Quid de l'opinion des familles des patients? Ne sont-elles pas concernées par ce type de pratique? Comment ces personnes trouvent-elles des repères ou des déterminants pour alerter les soignants de l'état de santé de leurs proches ? Notre recherche s'est centrée sur ce questionnement. En effet, cette problématique nous apparaît fondamentale, car potentiellement explicative d'une incompréhension entre soignants et familles de patients : les déterminants d'un état nécessitant une hospitalisation sont-ils les mêmes entre la famille d'un patient et son psychiatre? Il ne s'agit pas ici de mettre en question le jugement clinique des professionnels, mais, bien au contraire, de comprendre sur quels critères et déterminants se basent les familles de patients et le grand public, afin d'optimiser la communication et l'accompagnement de ces personnes.

L'objectif de notre étude « clinique de l'hospitalisation involontaire » est donc de mettre en lumière les déterminants de la décision d'hospitalisation involontaire chez le grand public, peu représenté dans la littérature internationale. Les termes utilisés ne sont pas anodins, mais procèdent de la prise en considération, d'une part, de l'enquête menée par M. Anguis et al. (2001) qui met au jour la pluralité des représentations liées au vocabulaire de la santé mentale et, d'autre part, de la volonté de vulgariser ce vocabulaire afin de ne pas créer d'ambiguïté dans la compréhension des vignettes cliniques par les répondants. Nous avons donc choisi d'utiliser les termes « trouble psychiatrique » et « hospitalisation involontaire » pour notre projet de recherche. Pour notre échantillon, nous souhaitons contacter des personnes issues de la population générale, en essayant de l'équilibrer au mieux au vu des caractéristiques démographiques classiques (sexe, âge, profession, niveau d'étude). Une attention plus particulière sera portée aux personnes ayant déjà vécu une situation d'hospitalisation involontaire d'un proche. Pour la création des vignettes cliniques, nous nous sommes appuyées sur les recherches réalisées sur le sujet et sur les recommandations de la Haute autorité de santé (HAS, 2005) concernant l'hospitalisation involontaire d'une personne atteinte d'un trouble mental. En effet, la loi préconise pour l'hospitalisation à la demande d'un tiers (HDT) la présence de troubles mentaux, l'impossibilité à consentir à l'hospitalisation, la nécessité de soins immédiats avec une surveillance constante en milieu hospitalier et, pour l'hospitalisation d'office (HO), la présence de troubles mentaux, la nécessité de soins et l'atteinte à la sûreté des personnes ou à l'ordre public. Cependant, la loi 
ne définit pas la nécessité de soins, laissant les psychiatres se référer aux critères cliniques : évaluation du risque hétéro et auto-agressif, troubles du comportement ou de l'adaptation, exploration de la sphère cognitive et le contenu-cours de la pensée, évaluation de la capacité de consentir. Selon la Haute autorité de santé, pour indiquer une hospitalisation sans consentement, on se doit de rechercher un risque suicidaire, un risque d'atteinte à autrui, une prise d'alcool ou de toxique associée, un délire ou des hallucinations, des troubles de l'humeur, le degré d'incurie. Nous proposerons donc aux répondants de se prononcer sur des situations de décisions d'hospitalisation involontaire, décisions sous-tendues par différents critères : risque suicidaire, risque hétéro-agressif, adhésion au traitement. Nous ajouterons également l'avis de la famille, afin d'évaluer l'impact de ce type de critère dans l'évaluation des personnes issues du grand public.

Les résultats attendus devront laisser apparaître les critères de décision d'une hospitalisation involontaire pour le grand public au regard de ceux présentés dans la littérature internationale concernant les psychiatres et les personnels médicaux. Plus précisément, nous chercherons à savoir quels seront les facteurs prépondérants dans la décision d'hospitaliser un patient : le risque de comportement hétéro-agressif apparaîtra-t-il comme le critère déterminant dans l'acceptabilité de la décision d'hospitalisation involontaire ? Le risque auto-agressif ou le risque de suicide va-t-il être également prépondérant dans cette décision, d'autant plus quand il fait suite à un antécédent similaire dans la biographie du patient? L'adhésion au traitement, sans être un facteur déterminant dans la décision, aura-t-il un impact, i.e. la décision d'hospitalisation sera-t-elle mieux acceptée si le patient non seulement a un risque de comportement hétéro ou auto-agressif, mais refuse, en outre, de suivre le traitement qui lui est proposé ? Enfin, l'avis de la famille n'aura-t-il qu'un impact marginal dans cette décision?

Les résultats attendus devront permettre de savoir s'il existe ou non une adéquation entre les réponses du grand public et les critères utilisés par les psychiatres et autres personnels médicaux présents dans la littérature internationale.

\section{Conclusion}

Ce projet de recherche en psychologie clinique de la santé va permettre de faire émerger des déterminants de la décision d'hospitalisation involontaire. À travers une thématique clinique, nous avons choisi d'aborder notre recherche sous l'angle de l'analyse de 
l'acceptabilité de cette décision et de la mise en exergue de ses déterminants, sous-tendue par la perception du risque, domaine spécifiquement étudié en psychologie de la santé.

\section{Références bibliographiques}

Anguis M. et al., 2001, « La perception des problèmes de santé mentale : les résultats d'une enquête sur neuf sites ", ministère de l'Emploi et de la Solidarité, DREES, 116.

Appelbaum P. S., Robbins P. C., Monahan J., 2000, «Violence and Delusions : Data From the MacArthur Violence Risk Assessment Study », American Journal of Psychiatry, 157 : 566572.

Bailey J. E. et al., 1997, « Risk Factors for Violent Death of Women in the Home », Archives Internal Medicine, 14, 157 (7) : 777-782.

Bernardet P., 2003, « Danger et dangerosité dans les législations nationales de santé mentale et la jurisprudence des pays européens », in Mitrossili B., Gravier C., Louzoun S., Stylianidis M., «Comité européen : droit, éthique et psychiatrie », Cahiers Pollen, 11 (Santé mentale et droits de l'Homme en Europe. Actes des Journées d'études européennes, Athènes, 6-7 décembre 1996).

Black D. A. et al., 1999, «Partner, Child Abuse Risk Factors Literature Review », National Network of Family Resiliency, National Network for Health (www.nnh.org/risk).

Coté G., 2001, « Les instruments d'évaluation du risque de comportements violents : mise en perspective critique », Criminologie, 34 (1) : 31-45.

Engleman N. B. et al., 1998, «Clinicians' Decision Making about Involuntary Commitment », Psychiatric Services, 49 : 941-945.

Gardner W. et al., 1996, «Clinical Versus Actuarial Predictions of Violence in Patients with Mental Illnesses », Journal of Consulting and Clinical Psychology, 64 : 602-609.

Haute autorité de santé, 2005, Modalités de prise de décision concernant l'indication en urgence d'une hospitalisation sans consentement d'une personne présentant des troubles mentaux.

Heise L., 1998, «Violence against Women : an Integrated Ecological Framework », Violence Against Women, 4 : 262-290.

Johnson D. A. W. et al., 1983, «The Discontinuance of Maintenance Neuroleptic Therapy in Chronic Schizophrenic Patients : Drug and Social Consequences », Acta Psychiatrica Scandinavica, $67: 339-352$ 
Kalunian D. A. et al., 1990, «Violence by Geriatric Patients Who Need Psychiatric Hospitalization », Journal of Clinical Psychiatry, 51 :340-343.

Kouabenan D. R. et al., 2006, Psychologie du risque. Identifier, évaluer, prévenir, Paris, De Boeck Université.

Lepping, P. et al., 2004, «Attitudes of Mental Health Professionals and Lay-People towards Involuntary Admission and Treatment in England and Germany - a Questionnaire Analysis », European Psychiatry, 19: 91-95.

Link B. G. et al., 1992, « The Violent and Illegal Behavior of Mental Patients Reconsidered », American Sociological Review, 57 : 275-292

Link B. G., Stueve A., Phelan J., 1998, « Psychotic Symptoms and Violent Behaviours : Probing the Components of "Threat/Control-Override" Symptoms ", Social Psychiatry and Psychiatric Epidemiology, 33 (1) : 55-60.

Luchins D. J. et al., 2004, « Psychiatrists' Attitudes toward Involuntary Hospitalization », Psychiatric Services, 55 : 1058-1060.

McCauley J. et al., 1995, "The "Battering Syndrome" : Prevalence and Clinical Characteristics of Domestic Violence in Primary Health Care Internal Medicine Practices », Annals of Internal Medicine, 123 : 737-746.

McNiel D. E. et al., 1992, « The Role of Violence in Decisions about Hospitalization from the Psychiatric Emergency Room », American Journal of Psychiatry, 148 : 1317-1321.

Monahan J., 1981, « The Clinical Prediction of Violent Behavior », DHHS Publication ADM, 8992, Rockville, National Institute of Mental Health.

Monahan J., 1992, « Mental Disorder and Violent Behavior Perceptions and Evidence », American Psychologist, 47 : 511-521.

Newhill, C. E. et al., 1995, « Characteristics of Violence in the Community by Female Patients Seen in a Psychiatric Emergency Service », Psychiatric Services, 46 : 785-789.

Swanson J. W. et al., 1990, Violence and Psychiatric Disorder in the Community : Evidence from the Epidemiologic Catchment Area Surveys », Hospital \& Community Psychiatry, 41 : 761-70.

Swanson J. W., 1994, « Mental Disorder, Substance Abuse and Community Violence : An Epidemiological Approach », in Monahan J., Steadman H., Violence and Mental Disorder. Developments in Risk Assessment, Chicago, University of Chicago Press.

Tardiff K., 1996, Assessment and Management of Violent Patients, Washington, American Psychiatric Press. 
Van Gijseghem H., 1996, «L'évaluation du présumé agresseur sexuel d'enfants », in Aubut J., Le Rôle du témoin expert, Montréal, Chenelière and Mac Graw-Hill.

Yesavage J. A. et al., 1983, «Family Conflict, Psychopathology and Dangerous Behaviour by Schizophrenic Inpatients », Psychiatry Research, $8: 271-280$. 